\title{
Existence and multiplicity of solutions to elliptic equations of fourth order on compact manifolds.
}

\author{
Mohammed Benalili \\ Communicated by Terence Tao, received March 28, 2008.
}

\begin{abstract}
This paper deals with a fourth order elliptic equation on compact Riemannian manifolds, the function $f$ involved in the nonlinearity is of changing sign which makes the analysis more difficult than the case where $f$ is of constant sign. We prove the multiplicity of solutions in the subcritical case which is the subject of the first theorem. In the second one we establish the existence of solutions to the equation with critical Sobolev growth.
\end{abstract}

\section{Contents}

1. Introduction

2. Preliminaries

3. A priori estimates 211

4. Subcritical case 218

5. Critical case 221

References $\quad 224$

\section{Introduction}

Let $(M, g)$ be a Riemannian compact smooth $n$ - manifold $n \geq 5$ with the metric $g$, we let $H_{2}^{2}(M)$ be the standard Sobolev space which is the completion of the space

$$
C_{2}^{2}(M)=\left\{u \in C^{\infty}(M):\|u\|_{2,2}<+\infty\right\}
$$

with respect to the norm $\|u\|_{2,2}=\sum_{l=0}^{2}\left\|\nabla^{l} u\right\|_{2}$.

2000 Mathematics Subject Classification. Primary 58J05.

Key words and phrases. Elliptic equation of fourth order, Critical Sobolev exponent. 
Let $H_{2}$ be the space $H_{2}^{2}$ endowed with the equivalent norm

$$
\|u\|_{H_{2}}=\left(\|\Delta u\|_{2}^{2}+\|\nabla u\|_{2}^{2}+\|u\|_{2}^{2}\right)^{\frac{1}{2}} \text {. }
$$

where, $\Delta(u)=-\operatorname{div}(\nabla u)$, denotes the Riemannian Laplacian.

First we establish the existence of at least two solutions of the subcritical equation

$$
\Delta^{2} u+\nabla^{i}\left(a(x) \nabla_{i} u\right)+h(x) u=f(x)|u|^{q-2} u
$$

where $2<q<N$. Next we investigate solutions of the critical equation

$$
\Delta^{2} u+\nabla^{i}\left(a(x) \nabla_{i} u\right)+h(x) u=f(x)|u|^{N-2} u
$$

where $a, h$ and $f$ are smooth functions on $M$ and $N=\frac{2 n}{n-4}$ is the critical exponent.

The function $f$ involved in the nonlinearity is of changing sign which makes the analysis more difficult than the case where $f$ is of constant sign.

The equation (1.1) has a geometric roots, in fact while the conformal Laplacian

$$
L_{g}(u)=\Delta u+\frac{n-2}{4(n-1)} R u
$$

where $R$ is the scalar curvature of the metric $g$, is associated to the scalar curvature; the Paneitz operator as discovered by Paneitz $([\mathbf{1 0}])$ on 4-dimension manifolds and extended by Branson $([\mathbf{3}])$ to higher dimensions $(n \geq 5)$ reads as

$$
P B_{g}(u)=\Delta^{2} u+\operatorname{div}\left(-\frac{(n-2)^{2}+4}{2(n-1)(n-2)} R . g+\frac{4}{n-2} R i c\right) d u+\frac{n-4}{2} Q^{n} u
$$

where Ric is the Ricci curvature of $g$ and where

$$
Q^{n}=\frac{1}{2(n-1)} \Delta R+\frac{n^{3}-4 n^{2}+16 n-16}{8(n-1)^{2}(n-2)^{2}} R^{2}-\frac{2}{(n-2)^{2}}|R i c|^{2}
$$

is associated to the notion of $Q$-curvature, good references on the subject are Chang $([5])$ and Chang-Yang $([\mathbf{6}])$. When the manifold $(M, g)$ is Einstein, the Paneitz-Branson operator has constant coefficients. It expresses as

$$
P B_{g}=\Delta^{2} u+\alpha \Delta u+a u
$$

with

$$
\alpha=\frac{n^{2}-2 n-4}{2 n(n-1)} R \text { and } a=\frac{(n-4)\left(n^{2}-4\right)}{16 n(n-1)^{2}} R^{2}
$$

and this operator is a special case of what it is usually referred as a Paneitz- Branson type operator with constant coefficients.

Since 1990 many results have been established for precise functions $a, h$ and $f$. D.E. Edmunds, D. Fortunato, E. Jannelli ([8]) proved for $n \geq 8$ that if $\lambda \in\left(0, \lambda_{1}\right)$, with $\lambda_{1}$ is the first eigenvalue of $\Delta^{2}$ on the euclidean open ball $B$, the problem

$$
\left\{\begin{array}{c}
\Delta^{2} u-\lambda u=u|u|^{\frac{8}{n-4}} \text { in } B \\
u=\frac{\partial u}{\partial n}=0 \text { on } \partial B
\end{array}\right.
$$

has a non trivial solution. 
In 1995, R. Van der Vorst ([12]) obtained the same results as D.E. Edmunds, D. Fortunato, E. Jannelli. when applied to the problem

$$
\left\{\begin{array}{c}
\Delta^{2} u-\lambda u=u|u|^{\frac{8}{n-4}} \text { in } \Omega \\
u=\Delta u=0 \text { on } \partial \Omega
\end{array}\right.
$$

where $\Omega$ is an open bounded set of $R^{n}$ and moreover he showed that the solution is positive

In ([7]) D.Caraffa studied the equation (1.1) on compact manifolds in the case $f(x)=$ constant; and in the particular case where the functions $a(x)$ and $h(x)$ are precise constants she obtained the existence of positive regular solutions.

In the case of second order equation related to the prescribed scalar curvature, that is

$$
\Delta u+\frac{n-2}{4(n-1)} R u=f u^{2^{*}-1}
$$

where $2^{*}=\frac{2 n}{n-2}$, A. Rauzy [11] stated, in the case where the scalar curvature $R$ of the manifold $(M, g)$ is a negative constant and $f$ is a changing sign function, the following results.

Let $f$ be a $C^{\infty}$ function on $M, f^{-}=-\inf (f, 0), f^{+}=\sup (f, 0)$ and

$$
\lambda_{f}=\inf _{u \in A} \frac{\int_{M}|\nabla u|^{2} d v_{g}}{\int_{M} u^{2} d v g}
$$

where $A=\left\{u \in H_{1}^{2}(M), u \geq 0, \quad u \not \equiv 0\right.$ s.t. $\left.\int_{M} f^{-} u d v_{g}=0\right\}$, and $\lambda_{f}=+\infty$ if $A=\phi$.

THEOREM 1. Let $(M, g)$ be a smooth manifold with constant scalar curvature $R<0$ and let $f$ be a smooth changing sign function on $M$. Suppose that there exists a constant $C>0$ which depends only on $\frac{f^{-}}{\int_{M} f^{-} d v_{g}}$ such that if $f$ fulfills the following conditions

(1) $|R|<\frac{4(n-1)}{n-2} \lambda_{f}$

(2) $\frac{\sup f^{+}}{\int f-d v_{g}}<C$.

Then, the equation (1.3) admits a positive solution.

THEOREM 2. Let $(M, g)$ be a smooth manifold with constant scalar curvature $R<0$ and let $f$ be a smooth changing sign function on $M$. Suppose that there exists a constant $C>0$ which depends only on $\frac{f^{-}}{\int_{M} f^{-} d v_{g}}$ such that if $f$ fulfills the following conditions

(1) $|R|<\frac{4(n-1)}{n-2} \lambda_{f}$

(2) $\frac{\sup f^{+}}{\int f-d v_{g}}<C$

(3) $\sup _{M} f>0$.

Then the subcritical equation $\left.\Delta_{g} u+R u=f u^{q-1}, q \in\right] 2,2^{*}[$ admits two nontrivial distinct solutions.

More recently [2] the authors have extended the work of Rauzy to the case of the so called generalized prescribed scalar curvature type equation

$$
\Delta_{p} u+a u^{p-1}=f u^{p^{*}-1}
$$


where $p^{*}=\frac{n p}{n-p}, \Delta_{p} u=-\operatorname{div}\left(|\nabla u|^{p-2} \nabla u\right)$ is the $p$-Laplacian operator on a compact manifold $M$ of dimension $n \geq 3$, with negative scalar curvature, $p \in(1, n)$, $u \in H_{1}^{p}(M)$ is a positive function, $f$ is a changing sign function and $a$ is a negative constant. Let

$$
\lambda_{f}=\inf _{u \in A} \frac{\int_{M}|\nabla u|^{p} d v_{g}}{\int_{M} u^{p} d v g}
$$

where $A=\left\{u \in H_{1}^{p}(M), u \geq 0, u \neq \equiv\right.$ s.t. $\left.\int_{M} f^{-} u d v_{g}=0\right\}$, and $\lambda_{f}=+\infty$ if $A=\phi$

Theorem 3. (Critical case) There is a constant $C>0$ which depends only on $f^{-} /\left(\int f^{-} d v_{g}\right)$ such that if $f \in C^{\infty}$ on $M$ fulfills the following conditions

(1) $|a|<\lambda_{f}$

(2) $\left(\operatorname{supf}^{+} / \int f^{-} d v_{g}\right)<C$.

Then the equation (1.4) has a positive solution of class $C^{1, \alpha}(M)$.

THEOREM 4. (Subcritical case) For every $C^{\infty}$ - function on $M$ there is a constant $C>0$ which depends only on $f^{-} /\left(\int f^{-} d v_{g}\right)$ such that if $f$ fulfills the following conditions

(1) $|a|<\lambda_{f}$

(2) $\left(\operatorname{supf} f^{+} / \int f^{-} d v_{g}\right)<C$

(3) $\sup f>0$.

Then the subcritical equation

$$
\left.\Delta_{p} u+a u^{p-1}=f u^{q-1} \quad q \in\right] p, p^{*}[
$$

has at least two non trivial positive solutions of class $C^{1, \alpha}(M)$.

For $a, f, C^{\infty}$-functions $M$, we let

$$
\lambda_{a, f}=\inf _{u \in A} \frac{\int_{M}(\Delta u)^{2} d v_{g}-\int_{M} a|\nabla u|^{2} d v_{g}}{\int_{M} u^{2} d v g}
$$

where $A=\left\{u \in H_{2}, u \geq 0, u \neq 0\right.$ s. t. $\left.\int_{M} f^{-} u d v_{g}=0\right\}$, and

$$
\lambda_{a, f}=+\infty \quad \text { if } \quad A=\phi .
$$

Let $h$ be a smooth negative function on $M$, we consider the functional $F_{q}$ defined on $\mathrm{H}_{2}$ by

$$
F_{q}(u)=\|\Delta u\|_{2}^{2}-\int_{M} a|\nabla u|^{2} d v_{g}+\int_{M} h u^{2} d v_{g}-\int_{M} f|u|^{q} d v_{g}, \quad q \in(2, N] .
$$

In the case of fourth order elliptic equations on manifolds with changing sign right hand side, no work is done at least I know off. While we borrow ideas from the paper of Rauzy $([\mathbf{1 2}])$, our method is not an adaptation of that of Rauzy, since the behavior of fourth order operators differs from that of second order ones. It is essentially due to the structures of the spaces $H_{1}^{2}(M)$ and $H_{2}^{2}(M)$ : indeed if $u \in H_{1}^{2}(M)$ so does $|u|$ and the gradient of $|u|$ satisfies $|\nabla| u||=|\nabla u|$ and also the analysis on $H_{2}^{2}(M)$ is more complicated than on $H_{1}^{2}(M)$. In this paper we state the following results

TheOrem 5. Let $a, h$ be $C^{\infty}$ functions on $M$ with $h$ negative. For every $C^{\infty}$ function, $f$ on $M$ with $\int_{M} f^{-} d v_{g}>0$, there exists a constant $C>0$ which depends 
only on $\frac{f^{-}}{\int f^{-} d v_{g}}$ such that if $f$ satisfies the following conditions

(1) $|h(x)|<\lambda_{a, f}$

for any $x \in M$

(2) $\frac{\sup f^{+}}{\int f-d v_{g}}<C$

(3) $\sup _{M} f>0$,

then the subcritical equation

$$
\left.\Delta^{2} u+\nabla^{i}\left(a \nabla_{i} u\right)+h u=f|u|^{q-2} u, \quad q \in\right] 2, N[
$$

has at least two distinct solutions $u$ and $v$ satisfying $F_{q}(u)<0<F_{q}(v)$ and of class $C^{4, \alpha}$, for some $\alpha \in(0,1)$.

TheOREM 6. Let $a, h$ be $C^{\infty}$ functions on $M$ with $h$ negative. For every $C^{\infty}$ function $f$ on $M$ with $\int_{M} f^{-} d v_{g}>0$ there exists a constant $C>0$ which depends only on $\frac{f^{-}}{\int f^{-} d v_{g}}$ such that if $f$ satisfies the following conditions

(1) $|h(x)|<\lambda_{a, f} \quad$ for any $x \in M$

(2) $\frac{\sup f^{+}}{\int f-d v_{g}}<C$

the critical equation

$$
\Delta^{2} u+\nabla^{i}\left(a \nabla_{i} u\right)+h u=f|u|^{N-2} u
$$

has a solution of class $C^{4, \alpha}$, for some $\alpha \in(0,1)$, with negative energy.

To have applications to conformal geometry, we must obtain positive solutions but this is a difficult problem because of the lack of a maximum principle, This will be treated in a separated work.

If the set $A=\phi$, the condition (1) of Theorem 5 and 6 is fulfilled.

Suppose that $A \neq \phi$ and let $\mu=\inf _{u \in A} \frac{\int_{M}|\nabla u| d v_{g}}{\int_{M} u^{2} d v_{g}}$.

REMARK 1. We get smooth functions for which we have solutions by observing that $\int_{\{x \in M: f(x) \geqslant 0\}} d v_{g}<\left(K_{2}^{2}+\epsilon\right)\|h\|_{\infty}+A_{2}(\epsilon)+\mu\|a\|_{\infty}$ implies $\lambda_{a, f}>\|h\|_{\infty}$ ( See Lemma 2) where $\epsilon$ is any positive real number and $K_{2}, A_{2}(\epsilon)$ are the constants of the Sobolev inequality given by Lemma 1.

Let $B_{k, q}=\left\{u \in H_{2}:\|u\|_{q}^{q}=k\right\}$, where \|\|$_{q}$ denotes the $L^{q}$-norm, and put $\mu_{k, q}=\inf _{u \in B_{k, q}} F_{q}(u)$. The method used in this paper consists in the case of Theorem 5 , to show that the curve $k \rightarrow \mu_{k, q}$ is continuous as a function of the argument $k$, starts at 0 goes by a relative negative minimum, which is attained, and takes positive values for $k$ in some interval $l_{q}$ and finally goes to $-\infty$, to do so many a priori estimates are given, then we deduce the existence of two solutions of the subcritical equation, one of negative energy and the other of positive energy. For the proof of Thorem 6 , we show that the sequence of solutions of the subcritical equations, with negative energies,obtained in Theorem 5 is bounded in $H_{2}$ as $q$ tends to $N=\frac{2 n}{n-4}$, the critical Sobolev exponent. By classical arguments, we show that up to a subsequence $u_{q}$ converges weakly to a solution $u$ of the critical equation. After, we show that $u$ is of negative energy i.e. $u \not \equiv 0$. 


\section{Preliminaries}

Let $a, h$ be $C^{\infty}$ functions on $M$ with $h$ negative. We suppose without lost of generality that the Riemannian manifold $(M, g)$ is of volume equals to 1 . Since it is equivalent to solve the equation (1.1) with $f$ or $\alpha f$ ( $\alpha$ a real number $\neq 0$ ), we consider the functional $F_{q}$ defined on $\mathrm{H}_{2}$ by

$$
F_{q}(u)=\|\Delta u\|_{2}^{2}-\int_{M} a|\nabla u|^{2} d v_{g}+\int_{M} h u^{2} d v_{g}-\int_{M} f|u|^{q} d v_{g}, \quad q \in(2, N)
$$

and set

where $k$ is some constant. Let

$$
B_{k, q}=\left\{u \in H_{2}(M),\|u\|_{q}^{q}=k\right\}
$$

$$
\mu_{k, q}=\inf _{u \in B_{k, q}} F_{q}(u)
$$

we state

Proposition 1. The infimum $\mu_{k, q}$ is achieved. Futhermore any minimizer of the functional $F_{q}$ is of class $C^{4, \alpha}, \alpha \in(0,1)$.

Proof. We have

$$
\begin{gathered}
F_{q}(u) \geq\|\Delta u\|_{2}^{2}-\left\|a_{+}\right\|_{\infty}\|\nabla u\|_{2}^{2}+k^{\frac{2}{q}} \min _{x \in M} h(x) \\
-k \max _{x \in M} f(x) .
\end{gathered}
$$

where $a_{+}(x)=\max [a(x), 0]$ and $\|\cdot\|_{\infty}$ is the supremum norm.

The following formula is well known on compact manifolds

$$
\begin{aligned}
\left\|\nabla^{2} u\right\|_{2}^{2} & \leq\|\Delta u\|_{2}^{2}-\int_{M} R i c_{i j} \nabla u_{i} \nabla u_{j} d v_{g} \\
& \leq\|\Delta u\|_{2}^{2}+\beta\|\nabla u\|_{2}^{2} .
\end{aligned}
$$

where $\beta$ is some constant. As it is shown in ([1] p.93), for any $\eta>0$, there exists a constant $C(\eta)$ depending on $\eta$ such that

$$
\|\nabla u\|_{2}^{2} \leq \eta\left\|\nabla^{2} u\right\|_{2}^{2}+C(\eta)\|u\|_{2}^{2}
$$

Plugging (2.2) in (2.3), we get

$$
\|\nabla u\|_{2}^{2} \leq \eta\|\Delta u\|_{2}^{2}+\eta \beta\|\nabla u\|_{2}^{2}+C(\eta)\|u\|_{2}^{2}
$$

and choosing $\eta$ such that $\eta \beta \leq \frac{1}{2}$, we obtain

$$
\|\nabla u\|_{2}^{2} \leq 2 \eta\|\Delta u\|_{2}^{2}+2 C(\eta)\|u\|_{2}^{2} .
$$

The inequality (2.1) reads then

$$
\begin{gathered}
F_{q}(u) \geq\|\Delta u\|_{2}^{2}\left(1-2 \eta\left\|a_{+}\right\|_{\infty}\right) \\
+k^{\frac{2}{q}}\left(\min _{x \in M} h(x)-2 C(\eta)\left\|a_{+}\right\|_{\infty}\right)-k \max _{x \in M} f(x)
\end{gathered}
$$

and then, with $\eta$ small enough, we have

$$
1-2 \eta\left\|a_{+}\right\|_{\infty}=\alpha>0
$$

so

$$
F_{q}(u) \geq \alpha\|\Delta u\|_{2}^{2}+C_{1}
$$


where $\alpha$ is some positive constant and $C_{1}$ is a constant independent of $u$. Let $\left(u_{j}\right)$ be a minimizing sequence of the functional $F_{q}$ in $B_{k, q}$; so for $j$ sufficiently large $F_{q}\left(u_{j}\right) \leq \mu_{k, q}+1$ and by $(2.6)$, we get

$$
\left\|\Delta u_{j}\right\|_{2}^{2} \leq \frac{1}{\alpha}\left(\mu_{k, q}+1-C_{1}\right) .
$$

By formula (2.5) and the fact

$$
\left\|u_{j}\right\|_{2}^{2} \leq k^{\frac{2}{q}}
$$

we obtain that $\left\|\nabla u_{j}\right\|_{2}^{2}$ is bounded. It follows that the sequence $\left(u_{j}\right)$ is bounded in $H_{2}$. Consequently $u_{j}$ converges weakly in $H_{2}$, the compact embedding of $H_{2}$ in $L^{q}$ and the unicity of the weak limit allow us to claim that there is a subsequence of $\left(u_{j}\right)$ still denoted $\left(u_{j}\right)$ such that

$$
u_{j} \rightarrow u \text { strongly in } L^{s} \quad \text { for any } s<N
$$

$$
\nabla u_{j} \rightarrow \nabla u \text { strongly in } L^{2}
$$

and

$$
\|u\|_{H_{2}} \leq \liminf _{j}\left\|u_{j}\right\|_{H_{2}}
$$

Consequently

$$
\begin{gathered}
F_{q}(u)=\|\Delta u\|_{2}^{2}-\int_{M} a|\nabla u|^{2} d v_{g}+\int_{M} h u^{2} d v_{g}-\int_{M} f|u|^{q} d v_{g} \\
\leq \liminf _{j}\left\|\Delta u_{j}\right\|_{2}^{2}-\lim _{j} \int_{M} a\left|\nabla u_{j}\right|^{2} d v_{g}+\lim _{j} \int_{M} h u_{j}^{2} d v_{g}-\lim _{j} \int_{M} f\left|u_{J}\right|^{q} d v_{g} \\
=\lim _{J} F_{q}\left(u_{j}\right)=\mu_{k, q}
\end{gathered}
$$

and since clearly

$$
\|u\|_{q}^{q}=k
$$

we obtain that

$$
F_{q}(u)=\mu_{k, q} .
$$

So $u$ fulfills

$$
\begin{gathered}
\int_{M} \Delta u \cdot \Delta v d v_{g}-\int_{M} a(x) \nabla^{i} u \cdot \nabla_{i} v d v_{g}+\int_{M} h(x) u v d v_{g} \\
-\frac{q}{2} \int_{M} f(x)|u|^{q-2} u v d v_{g}=\lambda_{k, q} \int_{M}|u|^{q-2} u v d v_{g}
\end{gathered}
$$

for any $v \in H_{2}$; where $\lambda_{k, q}$ is the Lagrange multiplier and $u$ is a weak solution of the equation

$$
\Delta^{2} u+\nabla^{i}\left(a \nabla_{i} u\right)+h u=\left(\lambda_{k, q}+\frac{q}{2} f\right)|u|^{q-2} u .
$$

Using the bootstrap method, we show that $u \in L^{s}(M)$ for any $s<N$, so $P(u)=\Delta^{2} u+\nabla^{i}\left(a \nabla_{i} u\right)+h u \in L^{s}(M)$ for any $s<N$ and since $P$ is a fourth order elliptic operator, it follows by a well known regularity theorem that $P(u) \in C^{0, \alpha}(M)$ for some $\alpha \in(0,1)$. Then $u \in C^{4, \alpha}(M)$.

Proposition 2. $\mu_{k, q}$ is continuous as a function of the argument $k$. 
Proof. For any $k, l \in R^{+}$, let $u$ and $v$ be two functions of norm 1 in $L^{q}$ such that $F_{q}\left(k^{\frac{1}{q}} u\right)=\mu_{k, q}$ and $F_{q}\left(l^{\frac{1}{q}} v\right)=\mu_{l, q}$.

Then

$$
\begin{gathered}
\mu_{l, q}-\mu_{k, q}=F_{q}\left(l^{\frac{1}{q}} v\right)-F_{q}\left(k^{\frac{1}{q}} v\right)+F_{q}\left(k^{\frac{1}{q}} v\right)-\mu_{k, q} \\
=F_{q}\left(k^{\frac{1}{q}} v\right)-\mu_{k, q} \\
+\left(l^{\frac{2}{q}}-k^{\frac{2}{q}}\right)\left(\|\Delta v\|_{2}^{2}-\int_{M} a|\nabla v|^{2} d v_{g}+\int_{M} h v^{2} d v_{g}\right) \\
-(l-k) \int_{M} f|v|^{q} d v_{g} .
\end{gathered}
$$

On the other hand, we have

i.e.

$$
\begin{gathered}
\mu_{l, q}=F_{q}\left(l^{\frac{1}{q}} v\right)=l^{\frac{2}{q}}\left(\|\Delta v\|_{2}^{2}-\int_{M} a|\nabla v|^{2} d v_{g}+\int_{M} h v^{2} d v_{g}\right)-l \int_{M} f|v|^{q} d v_{g} \\
\leq F_{q}\left(l^{\frac{1}{q}}\right)=l^{\frac{2}{q}} \int_{M} h d v_{g}-l \int_{M} f d v_{g}
\end{gathered}
$$

$$
\begin{gathered}
\|\Delta v\|_{2}^{2}-\int_{M} a|\nabla v|^{2} d v_{g}+\int_{M} h v^{2} d v_{g} \leq \\
\int_{M} h d v_{g}-l^{1-\frac{2}{q}} \int_{M} f d v_{g}+l^{1-\frac{2}{q}} \int_{M} f|v|^{q} d v_{g} .
\end{gathered}
$$

Since $\|v\|_{q}^{q}=1$, it follows that the term $\int_{M} f|v|^{q} d v_{g}$ is bounded for any $l$ in a neighborhood of $k$ and so the term $\|\Delta v\|_{2}^{2}-\int_{M} a|\nabla v|^{2} d v_{g}+\int_{M} h v^{2} d v_{g}$ is upper bounded. Also since $\mu_{l, q}$ is lower bounded, it follows that $\|\Delta v\|_{2}^{2}-\int_{M} a|\nabla v|^{2} d v_{g}+$ $\int_{M} h v^{2} d v_{g}$ is bounded in a neighborhood of $k$.

Consequently

$$
\liminf _{l \rightarrow k}\left(\mu_{l, q}-\mu_{k, q}\right) \geq \liminf _{l \rightarrow k}\left(F_{q}\left(k^{\frac{1}{q}} v\right)-\mu_{k, q}\right)
$$

and by the definition of $\mu_{k, q}$, we get

$$
\liminf _{l \rightarrow k}\left(\mu_{l, q}-\mu_{k, q}\right) \geq 0 \text {. }
$$

By writing

we get

$$
\begin{gathered}
\mu_{l, q}-\mu_{k, q}=\mu_{l, q}-F_{q}\left(l^{\frac{1}{q}} u\right)+F_{q}\left(l^{\frac{1}{q}} u\right)-F_{q}\left(k^{\frac{1}{q}} u\right) \\
=\mu_{l, q}-F_{q}\left(l^{\frac{1}{q}} u\right) \\
+\left(l^{\frac{2}{q}}-k^{\frac{2}{q}}\right)\left(\|\Delta u\|_{2}^{2}-\int_{M} a|\nabla u|^{2} d v_{g}+\int_{M} h u^{2} d v_{g}\right) \\
-(l-k) \int_{M} f|u|^{q} d v_{g}
\end{gathered}
$$

$$
\lim _{l \rightarrow k} \sup \left(\mu_{l, q}-\mu_{k, q}\right) \leq 0
$$

and taking into account of (2.8), we obtain

$$
\lim _{l \rightarrow k} \mu_{l, q}=\mu_{k, q} .
$$




\section{A priori estimates}

First, we quote the following Lemma due to Djadli-Hebey-Ledoux and improved by Hebey $[\mathbf{9}]$.

Lemma 1. Let $M$ be a Riemannian compact manifold with dimension $n \geq 5$. For any $\epsilon>0$ there is a constant $A_{2}(\epsilon)$ such that for any $u \in H_{2} \quad\|u\|_{N}^{2} \leq$ $K_{2}^{2}(1+\epsilon)\|\Delta u\|_{2}^{2}+A_{2}(\epsilon)\|u\|_{2}^{2}$ with $K_{2}^{-2}=\pi^{2} n(n-4)\left(n^{2}-4\right) \Gamma\left(\frac{n}{2}\right)^{\frac{4}{n}} \Gamma(n)^{-\frac{4}{n}}$.

Suppose that the set $A=\left\{u \in H_{2}, u \neq \equiv\right.$ s. t. $\left.\int_{M} f^{-}|u| d v_{g}=0\right\}$ is non empty.

LeMma 2. If $\int_{\{x \in M: f(x) \geqslant 0\}} d v_{g}$ as a function of the variable $f$ tends to $0, \lambda_{a, f}$ goes to $+\infty$. In particular the condition $\int_{\{x \in M: f(x) \geqslant 0\}} d v_{g}<K_{2}^{2}(1+\epsilon)\|h\|_{\infty}+$ $A_{2}(\epsilon)+\mu\|a\|_{\infty}$ implies that $\lambda_{a, f}>\|h\|_{\infty}$.

Proof. For any $u \in A$, we obtain by applying successively the Hölder inequality and the Sobolev one given by Lemma 1,

$$
\begin{gathered}
\int_{\{x \in M: f(x) \geqslant 0\}} u^{2} d v_{g} \leq\left(\int_{\{x \in M: f(x) \geqslant 0\}}|u|^{N} d v_{g}\right)^{\frac{2}{N}}\left(\int_{\{x \in M: f(x) \geqslant 0\}} d v_{g}\right)^{1-\frac{2}{N}} \\
=\left(\int_{M}|u|^{N} d v_{g}\right)^{\frac{2}{N}}\left(\int_{\{x \in M: f(x) \geqslant 0\}} d v_{g}\right)^{\frac{4}{n}} \\
\leq\left(K_{2}^{2}(1+\epsilon)\|\Delta u\|_{2}^{2}+A_{2}(\epsilon)\|u\|_{2}^{2}\right)\left(\int_{\{x \in M: f(x) \geqslant 0\}} d v_{g}\right)^{\frac{4}{n}}
\end{gathered}
$$

So

$$
\left(\int_{\{x \in M: f(x) \geqslant 0\}} d v_{g}\right)^{-\frac{4}{n}} \leq K_{2}^{2}(1+\epsilon) \lambda_{a, f}+A_{2}(\epsilon)+\inf _{x \in A} \frac{\int_{M} a(x)|\nabla u|^{2} d v_{g}}{\|u\|_{2}^{2}}
$$

and letting $\mu=\inf _{x \in A} \frac{\int_{M}|\nabla u|^{2} d v_{g}}{\|u\|_{2}^{2}}$, we get that

$$
\lambda_{a, f} \geqslant \frac{1}{K_{2}^{2}(1+\epsilon)}\left(\left(\int_{\{x \in M: f(x) \geqslant 0\}} d v_{g}\right)^{-\frac{4}{n}}-A_{2}(\epsilon)-\mu\|a\|_{\infty}\right)
$$

where $\|a\|_{\infty}=\sup _{x \in M}|a(x)|$.

Hence if $\int_{\{x \in M: f(x) \geqslant 0\}} d v_{g}$ tends to 0 as a function of the variable $f, \lambda_{a, f}$ goes to $+\infty$.

Denote also by $\|h\|_{\infty}=\sup _{x \in M}|h(x)|$ the supremum norm.

As in $[\mathbf{1 1}]$, we define the quantities,

$$
\lambda_{a, f, \eta, q}=\inf _{u \in A(\eta, q)} \frac{\|\Delta u\|_{2}^{2}-\int_{M} a|\nabla u|^{2} d v g}{\|u\|_{2}^{2}}
$$

with

$$
A(\eta, q)=\left\{u \in H_{2}:\|u\|_{q}=1, \int_{M} f^{-}|u|^{q} d v_{g}=\eta \int_{M} f^{-} d v_{g}\right\}
$$

for a real $\eta>0$, 
and

$$
\lambda_{a, f, \eta, q}^{\prime}=\inf _{u \in A^{\prime}(\eta, q)} \frac{\|\Delta u\|_{2}^{2}-\int_{M} a|\nabla u|^{2} d v g}{\|u\|_{2}^{2}}
$$

where

$$
A^{\prime}(\eta, q)=\left\{u \in H_{2}:\|u\|_{q}^{q}=1, \int_{M} f^{-}|u|^{q} d v_{g} \leq \eta \int_{M} f^{-} d v_{g}\right\} .
$$

Now, we will study $\lambda_{a, f, \eta, q}$, to do so, we distinguish ( as it is done in [11]) the case where the set $\{x \in M: f(x) \geq 0\}$ is of positive measure with respect to Riemannian measure and the case where the set is negligible and $\sup _{x \in M} f=0$.

Case $f^{+}>0$.

Claim 1. For any real $\eta>0$, the set $A(\eta, q)$ is not empty .

Indeed, the set $A^{\prime}(\eta, q)$ is not empty since it includes the set of functions $u \in H_{2}$ such that $\|u\|_{q}=1$ and with supports in the set $\left\{x \in M: f^{-}(x)<\eta \int_{M} f^{-} d v_{g}\right\}$. The same arguments as in [11] show that $\lambda_{a, f, \eta, q}^{\prime}$ is achieved by a function $v \in$ $A^{\prime}(\eta, q)$ and moreover $v$ satisfies $\int_{M} f^{-}|u|^{q} d v_{g}=\eta \int_{M} f^{-} d v_{g}$.

The following facts which are proved in [11], for the Laplacian operator remain valid in the case of the bi-Laplacian operator: $\lambda_{a, f, \eta, q}^{\prime}$ is a decreasing function with respect to $\eta$, bounded by $\lambda_{a, f}$ and $\lambda_{a, f, \eta, q}=\lambda_{a, f, \eta, q}^{\prime}$, so $\lambda_{a, f, \eta, q}$ is also a decreasing function with respect to $\eta$, and bounded by $\lambda_{a, f}$.

Lemma 3. For any $q \in] 2, N\left[, \lambda_{a, f, \eta, q}\right.$ goes to $\lambda_{a, f}$ whenever $\eta$ goes to zero.

Proof. $\lambda_{a, f, \eta, q}$ is attained by a family of functions labelled $v_{\eta, q}$. The functions $v_{\eta, q}$ indexed by $\eta$ are bounded in $H_{2}^{2}$ : since

$$
\left\|v_{\eta, q}\right\|_{2}^{2} \leq\left\|v_{\eta, q}\right\|_{q}^{2} \operatorname{Vol}(M)^{1-\frac{2}{q}}=1
$$

and

$$
\begin{aligned}
\left\|\Delta v_{\eta, q}\right\|_{2}^{2}- & \left\|a_{+}\right\|_{\infty}\left\|\nabla v_{\eta, q}\right\|_{2}^{2} \leq \lambda_{a, f, \eta, q}\left\|v_{\eta, q}\right\|_{2}^{2} \\
& \leq \lambda_{a, f}\left\|v_{\eta, q}\right\|_{2}^{2} \leq \lambda_{a, f} .
\end{aligned}
$$

By formula (2.5), for a well chosen $\varepsilon>0$, there is a constant $C(\varepsilon)>0$ such that

$$
\left\|\nabla v_{\eta, q}\right\|_{2}^{2} \leq 2 \varepsilon\left\|\Delta v_{\eta, q}\right\|_{2}^{2}+2 C(\varepsilon)\left\|v_{\eta, q}\right\|_{2}^{2}
$$

SO

$$
\begin{gathered}
\left\|\Delta v_{q, \eta}\right\|_{2}^{2} \leq \lambda_{a, f}+\left\|a_{+}\right\|_{\infty}\left\|\nabla v_{n, q}\right\|_{2}^{2} \\
\leq \lambda_{a, f}+2\left\|a_{+}\right\|_{\infty}\left(\varepsilon\left\|\Delta v_{\eta, q}\right\|_{2}^{2}+C(\varepsilon)\left\|v_{\eta, q}\right\|_{2}^{2}\right)
\end{gathered}
$$

and

$$
\left\|\Delta v_{q, \eta}\right\|_{2}^{2}\left(1-2 \varepsilon\left\|a_{+}\right\|_{\infty}\right) \leq \lambda_{a, f}+2\left\|a_{+}\right\|_{\infty} C(\varepsilon) .
$$

By choosing $\varepsilon>0$ small enough such that

$$
1-2 \varepsilon\left\|a_{+}\right\|_{\infty}>0
$$

we get that

$$
\left\|\Delta v_{q, \eta}\right\|_{2}^{2} \leq C^{\prime}\left(\lambda_{a, f},\left\|a_{+}\right\|_{\infty}, \varepsilon\right)
$$

where $C^{\prime}\left(\lambda_{a, f},\left\|a_{+}\right\|_{\infty}, \varepsilon\right)$ is a constant depending of $\lambda_{a, f},\left\|a_{+}\right\|_{\infty}, \varepsilon$.

$$
\left\|\nabla v_{q, \eta}\right\|_{2}^{2} \leq 2 \varepsilon C\left(\lambda_{a, f},\left\|a_{+}\right\|_{\infty}, \varepsilon\right)+2 C(\varepsilon) \leq C^{\prime}\left(\lambda_{a, f},\left\|a_{+}\right\|_{\infty}, \varepsilon\right) .
$$

Consequently the sequence $\left(v_{q, \eta}\right)_{\eta}$ is bounded in $\mathrm{H}_{2}$ and we have 


$$
\begin{gathered}
v_{q \eta} \longrightarrow v_{q} \text { weakly in } H_{2} . \\
v_{q \eta} \longrightarrow v_{q} \text { strongly in } H_{r}^{2}, \quad r=0,1 \\
v_{q \eta} \longrightarrow v_{q} \text { strongly in } L^{q}
\end{gathered}
$$

and

$$
\left\|\Delta v_{q}\right\|_{2}^{2} \leq \lim _{\eta \rightarrow 0} \inf \left\|\Delta v_{q \eta}\right\|_{2}^{2}
$$

Also

On the other hand

$$
\left\|v_{q}\right\|_{q}=1
$$

$$
\int_{M} f^{-}\left|v_{q \eta}\right|^{q} d v_{g}=\eta \int_{M} f^{-} d v_{g}
$$

so

$$
\int_{M} f^{-}\left|v_{q}\right|^{q} d v_{g}=0
$$

Hence

$$
v_{q} \in A
$$

and

$$
\begin{gathered}
\left\|v_{q}\right\|_{2}^{2} \lambda_{a, f} \leq\left\|\Delta v_{q}\right\|_{2}^{2}-\int_{M} a\left|\nabla v_{q}\right|^{2} d v_{g} \\
\leq \lim _{\eta \longrightarrow 0} \inf \left(\left\|\Delta v_{q \eta}\right\|_{2}^{2}-\int_{M} a\left|\nabla v_{q \eta}\right|^{2} d v_{g}\right)=\lim _{\eta \longrightarrow 0} \inf \left\|v_{q \eta}\right\|_{2}^{2}\left(\lambda_{a, f, q, \eta}\right)
\end{gathered}
$$

and since by construction

$$
\lambda_{a, f} \geq \lambda_{a, f, q, \eta}
$$

we get that

$$
\lim _{\eta \longrightarrow 0} \lambda_{a, f, q, \eta}=\lambda_{a, f} .
$$

Lemma 4. Let $\varepsilon>0$, there exists $\eta_{o}$ such that for any $\eta<\eta_{o}$, there is $q_{\eta}$ such that $\lambda_{a, f, q, \eta} \geq \lambda_{a, f}-\varepsilon$ for any $q>q_{\eta}$.

Proof. We proceed by contradiction. Suppose that there is a $\varepsilon_{o}>0$, such that for any $\eta$ there exists an $\eta_{o}<\eta$ and for any $q_{\eta o}$ there is $q>q_{\eta o}$ with $\lambda_{a, f, q, \eta}<\lambda_{f}-\varepsilon$. If $v_{q \eta}$ is the function in $H_{2}$ which achieves $\lambda_{a, f, q, \eta}$, then

$$
\lambda_{a, f, q, \eta}=\frac{\left\|\Delta v_{q \eta}\right\|_{2}^{2}-\int_{M} a\left|\nabla v_{q \eta}\right|^{2} d v_{g}}{\left\|v_{q \eta}\right\|_{2}^{2}}
$$

with $\left\|v_{q \eta}\right\|_{q}^{q}=1$. For a convenient $\eta$, we choose a sequence $q$ converging to $N$ such that

$$
\left\|\Delta v_{q \eta}\right\|_{2}^{2}-\int_{M} a\left|\nabla v_{q \eta}\right|^{2} d v_{g}<\lambda_{a, f}-\varepsilon_{o} .
$$

By the same argument as in the proof of Lemma 3 , we get that the sequence $v_{q \eta}$ indexed by $q$ is bounded in $H_{2}$ so up to a subsequence $v_{q \eta}$ converges weakly to $v_{\eta}$ in $H_{2}$ and strongly in $H_{r}^{2}, r=0,1$. Also we have

$$
\left\|\Delta v_{\eta}\right\|_{2}^{2} \leq \lim _{q \longrightarrow N} \inf \left\|\Delta v_{q \eta}\right\|_{2}^{2}
$$


and by the strong convergence in $H_{r}^{2}, r=0,1$, we get

$$
\left\|\Delta v_{\eta}\right\|_{2}^{2}-\int_{M} a\left|\nabla v_{\eta}\right|^{2} d v_{g}<\left(\lambda_{a, f}-\varepsilon_{o}\right)\left\|v_{\eta}\right\|_{2}^{2} .
$$

By the Sobolev inequality given in the Lemma 1 we have for any $\varepsilon_{1}>0$ there is a constant $A\left(\varepsilon_{1}\right)>0$ such that

$$
\begin{gathered}
1=\left\|v_{q \eta}\right\|_{q}^{2} \leq\left\|v_{q \eta}\right\|_{N}^{2} \quad(\text { since the manifold } M \text { is of volume } 1) \\
\leq K_{2}^{2}\left(1+\varepsilon_{1}\right)\left\|\Delta v_{q \eta}\right\|_{2}^{2}+A\left(\varepsilon_{1}\right)\left\|v_{q \eta}\right\|_{2}^{2} \\
\leq\left[K_{2}^{2}\left(1+\varepsilon_{1}\right) \lambda_{a, f}+A\left(\varepsilon_{1}\right)\right]\left\|v_{q \eta}\right\|_{2}^{2}++\left(K_{2}^{2}+\varepsilon_{1}\right)\left\|a_{+}\right\|_{\infty}\left\|\nabla v_{q \eta}\right\|_{2}^{2} \\
\leq\left[K_{2}^{2}\left(1+\varepsilon_{1}\right)\left(1+\left\|a_{+}\right\|_{\infty}\right) \lambda_{a, f}+A\left(\varepsilon_{1}\right)\right]\left\|v_{q \eta}\right\|_{H_{1}^{2}}^{2} .
\end{gathered}
$$

Consequently

$$
\left\|v_{\eta}\right\|_{2}^{2} \geq \frac{1}{\left.\left[K_{2}^{2}\left(1+\varepsilon_{1}\right)\left(1+\left\|a_{+}\right\|_{\infty}\right) \lambda_{a, f}+A\left(\varepsilon_{1}\right)\right]\right)} .
$$

As in $[\mathbf{1 1}]$ we can show that

$$
\int_{M}\left|v_{\eta}\right|^{N} d v_{g} \leq 1 \text { and } \int_{M} f^{-}\left|v_{\eta}\right|^{N} d v_{g} \leq \eta \int_{M} f^{-} d v_{g} .
$$

Consider the sequence of $\eta$ such that for any $q_{\eta}$, there is a $q>q_{\eta}$ with

$$
\lambda_{a, f, q, \eta} \leq \lambda_{a, f}-\varepsilon
$$

Now tending $\eta$ to 0 , if $v_{\eta}$ is the sequence corresponding to $\eta$ previously considered, $v_{\eta}$ is bounded in $\mathrm{H}_{2}$ and

$$
\left\|v_{\eta}\right\|_{2}^{2} \geq \frac{1}{\left.\left[K_{2}^{2}\left(1+\varepsilon_{1}\right)\left(1+\left\|a_{+}\right\|_{\infty}\right) \lambda_{a, f}+A\left(\varepsilon_{1}\right)\right]\right)} .
$$

so $v_{\eta}$ converges weakly to $v \neq 0$ in $H_{2}$ and strongly to $v$ in $H_{r}^{2}, r=0,1$ and $v$ satisfies

$$
\|\Delta v\|_{2}^{2}-\int_{M} a|\nabla v|^{2} d v_{g} \leq\left(\lambda_{a, f}-\varepsilon_{o}\right)\|v\|_{2}^{2} .
$$

On the other hand

$$
0 \leq \int_{M} f^{-}|v|^{N} d v_{g} \leq \lim _{\eta \rightarrow 0} \inf \int_{M} f^{-}\left|v_{\eta}\right|^{N} d v_{g} \leq \lim _{\eta \rightarrow 0} \eta \int_{M} f^{-} d v_{g}=0
$$

then $\int_{M} f^{-}|v| d v_{g}=0$ and $v$ belongs to the domain $A$ of definition of $\lambda_{a, f}$. Hence

$$
\lambda_{a, f} \leq \frac{\|\Delta v\|_{2}^{2}-\int_{M} a|\nabla v|^{2} d v_{g}}{\int_{M}|v|^{2} d v_{g}} .
$$

A contradiction with the inequality (3.1) and Lemma 4 is proved.

Case $f^{+}=0$.

In this case $\lambda_{a, f}$ is not defined so $\lambda_{a, f}=+\infty$. First, we give the lemma equivalent to Lemma 3

Lemma 5. Let $q \in] 2, N\left[\right.$. For any positive constant $R$, there exists $\eta_{o}$ such that for any $\eta<\eta_{o}, \quad \lambda_{a, f, \eta, q} \geq R$. 
ProOF. We argue by contradiction. It is easy to show that $\lambda_{a, f, q, \eta}$ is achieved by a function $v_{q \eta}$ in $H_{2}$ with $\left\|v_{q, \eta}\right\|_{q}=1$. Suppose that there is $\lambda_{a, f, \eta, q}$ bounded when $\eta$ goes to 0 . Then

$$
\begin{gathered}
\left\|\Delta v_{q, \eta}\right\|_{2}^{2}-\left\|a_{+}\right\|_{\infty}\left\|\nabla v_{q, \eta}\right\|_{2}^{2} \leq \frac{\left\|\Delta v_{q, \eta}\right\|_{2}^{2}-\left\|a_{+}\right\|_{\infty}\left\|\nabla v_{q, \eta}\right\|_{2}^{2}}{\left\|v_{q, \eta}\right\|_{2}^{2}} \\
\leq \lambda_{a, f, q, \eta}<+\infty
\end{gathered}
$$

and proceeding as in the proof of Lemma 3 we get that the sequence $v_{q \eta}$ indexed by $\eta$ is bounded in $\mathrm{H}_{2}$. Consequently the sequence $v_{q \eta}$ converges weakly to $v_{q}$ in $\mathrm{H}_{2}$ and converges strongly to $v_{q}$ in $H_{r}^{2}, r=0,1$, and strongly to $v_{q}$ in $L^{q}$ as $\eta$ goes to 0. $\int_{M} f^{-}\left|v_{q}\right|^{q} d v_{g}=0$ which implies that $v_{q}=0$ almost everywhere and $\left\|v_{q}\right\|_{q}=1$ which are in contradiction with each other.

Now we give an analogue to Lemma 4.

LEMMA 6. There exists an $\eta_{o}$ such that for any $\eta<\eta_{o}$ there is $q_{\eta}$ such that for any $q>q_{\eta}$ we have $\lambda_{a, f, q, \eta}>\|h\|_{\infty}$.

The proof of this lemma is similar to the previous ones so we omit it.

Let $\sigma>0$, any sufficient small real number, with the previous notations we obtain by using the lemmas quoted above the following

LEMMA 7. (1) Suppose that $\sup _{M} f>0$ and $\|h\|_{\infty}<\lambda_{a, f}$. There exists $\eta$ such that $\lambda_{a, f, q, \eta}-\|h\|_{\infty}=\varepsilon_{o}>0$.

Let $b=\frac{\left(1-2 \sigma\left\|a_{+}\right\|_{\infty}\right) \varepsilon_{o}}{\left[\left(\varepsilon_{o}+\|h\|_{\infty}+2\left\|a_{+}\right\|_{\infty} C(\sigma)\right) K_{2}^{2}(1+\varepsilon)+\left(1-2 \sigma\left\|a_{+}\right\|_{\infty}\right) A(\varepsilon)\right]}$

$\mu=\inf \left(b,\|h\|_{\infty}+2\left\|a_{+}\right\|_{\infty} C(\sigma)\right)$ and suppose that

$\frac{\sup _{M} f}{\int_{M} f-d v_{g}}<\frac{\mu \eta}{8\left(\|h\|_{\infty}+2\left\|a_{+}\right\|_{\infty} C(\sigma)\right)}$, where and $K_{2}^{2}, A(\epsilon)$ are the constants appearing in the Sobolev inequality given by Lemma 1. For any $q \in] 2, N[$ there exists a non empty interval $I_{q} \subset R^{+}$such that for every $u \in H_{2}$ with $L^{q}$-norm $k^{\frac{1}{q}}$ and $k \in I_{q}=\left[k_{1, q}, k_{2, q}\right]$ we have $F_{q}(u) \geq \frac{1}{2} \mu k^{\frac{2}{q}}$.

(2) Suppose that $\sup _{M} f=0$ and $\|h\|_{\infty}<\lambda_{a, f}$, there exists an interval $I_{q}=$ $\left[k_{1, q},+\infty\left[\right.\right.$ such that for any $k \in I_{q}$ and any $u \in H_{2}$ with $\|u\|_{q}^{q}=k$, we have $F_{q}(u) \geq \frac{1}{2} \mu k^{\frac{2}{q}}$.

Proof. Case: $f^{+}>0$.

Let $u \in H_{2}$ such that $\|u\|_{q}^{q}=k$.

Putting

$$
G_{q}(u)=\|\Delta u\|_{2}^{2}-\int_{M} a|\nabla u|^{2} d v_{g}+\int_{M} h u^{2} d v_{g}+\int_{M} f^{-}|u|^{q} d v_{g},
$$

we get

$$
G_{q}(u) \geq\|\Delta u\|_{2}^{2}-\left\|a_{+}\right\|_{\infty}\|\nabla u\|_{2}^{2}-\|h\|_{\infty}\|u\|_{2}^{2}+\int_{M} f^{-}|u|^{q} d v_{g}
$$

and taking account of (2.5), we obtain that for any suitable real $\sigma>0$, there is a constant $C(\sigma)>0$ such that

$$
G_{q}(u) \geq\left(1-2 \sigma\left\|a_{+}\right\|_{\infty}\right)\|\Delta u\|_{2}^{2}
$$


So if

$$
-\left(\|h\|_{\infty}+2 C(\sigma)\left\|a_{+}\right\|_{\infty}\right)\|u\|_{2}^{2}+\int_{M} f^{-}|u|^{q} d v_{g} .
$$

then

$$
\int_{M} f^{-}|u|^{q} d v g \geq \eta k \int_{M} f^{-} d v_{g}
$$

$$
\begin{gathered}
G_{q}(u) \geq\left(1-2 \sigma\left\|a_{+}\right\|_{\infty}\right)\|\Delta u\|_{2}^{2} \\
-\left(\|h\|_{\infty}+2 C(\sigma)\left\|a_{+}\right\|_{\infty}\right)\|u\|_{2}^{2}+\eta k \int_{M} f^{-} d v_{g}
\end{gathered}
$$

with $\sigma>0$ sufficiently small so that

$$
1-2 \sigma\left\|a_{+}\right\|_{\infty}>0 .
$$

Now since

$$
\|u\|_{2}^{2} \leq\|u\|_{q}^{\frac{2}{q}} \operatorname{Vol}(M)^{1-\frac{2}{q}}=k^{\frac{2}{q}}
$$

we get

$$
\begin{gathered}
G_{q}(u) \geq k^{\frac{2}{q}}\left[-\left(\|h\|_{\infty}+2\left\|a_{+}\right\|_{\infty} C(\sigma)\right)+\eta k^{1-\frac{2}{q}} \int_{M} f^{-} d v_{g}\right] \\
\geq k^{\frac{2}{q}}\left(\|h\|_{\infty}+2\left\|a_{+}\right\|_{\infty} C(\sigma)\right)\left(\frac{\eta k^{1-\frac{2}{q}}}{\|h\|_{\infty}+2\left\|a_{+}\right\|_{\infty} C(\sigma)} \int_{M} f^{-} d v_{g}-1\right)
\end{gathered}
$$

and choosing $k$ such that

$$
\frac{\eta k^{1-\frac{2}{q}}}{\|h\|_{\infty}+2\left\|a_{+}\right\|_{\infty} C(\sigma)} \int_{M} f^{-} d v_{g}-1 \geq 1
$$

that is

we obtain

$$
k \geq\left[2 \frac{\|h\|_{\infty}+2\left\|a_{+}\right\|_{\infty} C(\sigma)}{\eta \int_{M} f^{-} d v_{g}}\right]^{\frac{q}{q-2}}
$$

Let

$$
G_{q}(u) \geq k^{\frac{2}{q}}\left(\|h\|_{\infty}+2\left\|a_{+}\right\|_{\infty} C(\sigma)\right)
$$

$$
k_{1, q}=\left[2 \frac{\|h\|_{\infty}+2\left\|a_{+}\right\|_{\infty} C(\sigma)}{\eta \int_{M} f^{-} d v_{g}}\right]^{\frac{q}{q-2}} .
$$

In the case $\int_{M} f^{-}|u|^{q} d v_{g}<\eta k \int_{M} f^{-} d v_{g}$, we have

so

$$
\|\Delta u\|_{2}^{2}-\int_{M} a|\nabla u|^{2} d v_{g} \geq \lambda_{a, f, q, \eta}\|u\|_{2}^{2}
$$

$$
\begin{aligned}
& G_{q}(u) \geq \lambda_{a, f, \eta, q}\|u\|_{2}^{2}+\int_{M} h u^{2} d v_{g}+\int_{M} f^{-}|u|^{q} d v_{g} \\
& \geq\left(\lambda_{a, f, \eta, q}-\|h\|_{\infty}\right)\|u\|_{2}^{2}+\int_{M} f^{-}|u|^{q} d v_{g}
\end{aligned}
$$

by Lemma 4 and 6 there exists $\eta$ such that

$$
\lambda_{a, f, \eta, q}-\|h\|_{\infty}=\varepsilon_{o}>0 .
$$

Now, putting $\delta_{1}+\delta_{2}=\varepsilon_{o}$, where $\delta_{1}$ and $\delta_{2}$ are positive real numbers, and solving $\|u\|_{2}^{2}$ in (3.2), we get

$$
\|u\|_{2}^{2} \geq \frac{1}{\|h\|_{\infty}+2\left\|a_{+}\right\|_{\infty} C(\sigma)}\left[\left(1-2 \sigma\left\|a_{+}\right\|_{\infty}\right)\|\Delta u\|_{2}^{2}-G_{q}(u)+\int_{M} f^{-}|u|^{q} d v_{g}\right] .
$$


Consequently

$$
\begin{aligned}
& \left(1+\frac{\delta_{2}}{\|h\|_{\infty}+2\left\|a_{+}\right\|_{\infty} C(\sigma)}\right) G_{q}(u) \geq \delta_{1}\|u\|_{2}^{2} \\
& +\frac{\delta_{2}}{\|h\|_{\infty}+2\left\|a_{+}\right\|_{\infty} C(\sigma)}\left(1-2 \sigma\left\|a_{+}\right\|_{\infty}\right)\|\Delta u\|_{2}^{2}
\end{aligned}
$$

so

$$
G_{q}(u) \geq \frac{\delta_{1}\left(\|h\|_{\infty}+2\left\|a_{+}\right\|_{\infty} C(\sigma)\right)}{\|h\|_{\infty}+2\left\|a_{+}\right\|_{\infty} C(\sigma)+\delta_{2}}\|u\|_{2}^{2}+\frac{\delta_{2}\left(1-2 \sigma\left\|a_{+}\right\|_{\infty}\right)}{\|h\|_{\infty}+2\left\|a_{+}\right\|_{\infty} C(\sigma)+\delta_{2}}\|\Delta u\|_{2}^{2}
$$

and where $\sigma$ is sufficiently small and such that $1-2\left\|a_{+}\right\|_{\infty} \sigma>0$.

Or

$$
\begin{gathered}
G_{q}(u) \geq \frac{\delta_{2}\left(1-2 \sigma\left\|a_{+}\right\|_{\infty}\right)}{\left(\|h\|_{\infty}+2\left\|a_{+}\right\| \|_{\infty} C(\sigma)+\delta_{2}\right)\left(K_{2}^{2}+\varepsilon\right)} \\
\times\left[K_{2}^{2}(1+\varepsilon)\|\Delta u\|_{2}^{2}+\frac{\delta_{1}\left(\|h\|_{\infty}+2\left\|a_{+}\right\|_{\infty} C(\sigma)\right)\left(K_{2}^{2}+\varepsilon\right)}{\delta_{2}\left(1-2 \sigma\left\|a_{+}\right\|_{\infty}\right) A(\varepsilon)} A(\varepsilon)\|u\|_{2}^{2}\right]
\end{gathered}
$$

where for any fixed $\varepsilon>0, K_{2}^{2}$ denotes the best Sobolev constant in the embedding of $H_{2}^{2}\left(R^{n}\right)$ in $L^{q}\left(R^{n}\right)$.

Taking $\delta_{1}$ and $\delta_{2}$ such that

$$
\frac{\delta_{1}\left(\|h\|_{\infty}+2\left\|a_{+}\right\|_{\infty} C(\sigma)\right)\left(K_{2}^{2}+\varepsilon\right)}{\delta_{2}\left(1-2 \sigma\left\|a_{+}\right\|_{\infty}\right) A(\varepsilon)}=1
$$

we get

$$
\delta_{1}=\frac{\left(1-2 \sigma\left\|a_{+}\right\|_{\infty}\right) A(\varepsilon)}{\left(\|h\|_{\infty}+2\left\|a_{+}\right\|_{\infty} C(\sigma)\right) K_{2}^{2}(1+\varepsilon)+\left(1-2 \sigma\left\|a_{+}\right\|_{\infty}\right) A(\varepsilon)} \varepsilon_{o}
$$

and

$$
\delta_{2}=\frac{\left(\|h\|_{\infty}+2\left\|a_{+}\right\|_{\infty} C(\sigma)\right)\left(K_{2}^{2}+\varepsilon\right)}{\left(\|h\|_{\infty}+2\left\|a_{+}\right\|_{\infty} C(\sigma)\right) K_{2}^{2}(1+\varepsilon)+\left(1-2 \sigma\left\|a_{+}\right\|_{\infty}\right) A(\varepsilon)} \varepsilon_{o} .
$$

Consequently

$$
G_{q}(u) \geq \frac{\delta_{2}\left(1-2 \sigma\left\|a_{+}\right\|_{\infty}\right)}{\left(\|h\|_{\infty}+2\left\|a_{+}\right\|_{\infty} C(\sigma)+\delta_{2}\right)\left(K_{2}^{2}+\varepsilon\right)}\|u\|_{q}^{2}
$$

and since

$$
\begin{gathered}
\|h\|_{\infty}+2\left\|a_{+}\right\|_{\infty} C(\sigma)+\delta_{2}=\left(\|h\|_{\infty}+2\left\|a_{+}\right\|_{\infty} C(\sigma)\right) \\
\times\left[1+\frac{K_{2}^{2}(1+\varepsilon)}{\left(\|h\|_{\infty}+2\left\|a_{+}\right\|_{\infty} C(\sigma)\right) K_{2}^{2}(1+\varepsilon)+\left(1-2 \sigma\left\|a_{+}\right\|_{\infty}\right) A(\varepsilon)} \varepsilon_{o}\right] \\
=\frac{\left(\varepsilon_{o}+\|h\|_{\infty}+2\left\|a_{+}\right\|_{\infty} C(\sigma)\right) K_{2}^{2}(1+\varepsilon)+\left(1-2 \sigma\left\|a_{+}\right\|_{\infty}\right) A(\varepsilon)}{\left(\|h\|_{\infty}+2\left\|a_{+}\right\|_{\infty} C(\sigma)\right) K_{2}^{2}(1+\varepsilon)+\left(1-2 \sigma\left\|a_{+}\right\|_{\infty}\right) A(\varepsilon)}
\end{gathered}
$$

we get that

$$
G_{q}(u) \geq \frac{\left(1-2 \sigma\left\|a_{+}\right\|_{\infty}\right) \varepsilon_{o}}{\left[\left(\varepsilon_{o}+\|h\|_{\infty}+2\left\|a_{+}\right\|_{\infty} C(\sigma)\right) K_{2}^{2}(1+\varepsilon)+\left(1-2 \sigma\left\|a_{+}\right\|_{\infty}\right) A(\varepsilon)\right]} k^{\frac{2}{q}} .
$$

Letting

$$
b=\frac{\left(1-2 \sigma\left\|a_{+}\right\|_{\infty}\right) \varepsilon_{o}}{\left[\left(\varepsilon_{o}+\|h\|_{\infty}+2\left\|a_{+}\right\|_{\infty} C(\sigma)\right) K_{2}^{2}(1+\varepsilon)+\left(1-2 \sigma\left\|a_{+}\right\|_{\infty}\right) A(\varepsilon)\right]}
$$


we get

$$
\begin{gathered}
F_{q}(u)=G_{q}(u)-\int_{M} f^{+}|u|^{q} d v_{g} \\
\geq b k^{\frac{2}{q}}-\int_{M} f^{+}|u|^{q} d v_{g} \geq b k^{\frac{2}{q}}-k \sup f^{+}=k^{\frac{2}{q}}\left(b-k^{1-\frac{2}{q}} \sup f^{+}\right) .
\end{gathered}
$$

So if $\sup _{M} f>0$, let $\mu=\inf \left(b,\|h\|_{\infty}+2\left\|a_{+}\right\|_{\infty} C(\sigma)\right)$. For any $k \geq k_{1, q}$, we have

$$
F_{q}(u) \geq k^{\frac{2}{q}}\left(\mu-k^{1-\frac{2}{q}} \sup f\right)
$$

Now if we put $C_{q}=\frac{\eta}{8\left(\|h\|_{\infty}+2\left\|a_{+}\right\|_{\infty} C(\sigma)\right)} \mu$ and $\operatorname{suppose}$ that $\sup _{M} f \leq C_{q} \int_{M} f^{-}$, we obtain that the inequality is fulfilled provided that

$$
k \leq\left[\frac{4\left(\|h\|_{\infty}+2\left\|a_{+}\right\|_{\infty} C(\sigma)\right)}{\eta \int_{M} f^{-} d v_{g}}\right]^{\frac{q}{q-2}}=2^{\frac{q}{q-2}} k_{1, q} .
$$

and

provided that

$$
F_{q}(u) \geq \frac{1}{2} \mu k^{\frac{2}{q}}
$$

We put

$$
k \leq\left[\frac{\mu}{2 \sup f}\right]^{\frac{q}{q-2}}
$$

Case $f^{+}=0$.

$$
k_{2, q}=2^{\frac{q}{q-2}} k_{1, q}
$$

In this case, for any $k \geq k_{1, q}$,

$$
F_{q}(u) \geq \frac{1}{2} \mu k^{\frac{2}{q}}
$$

\section{Subcritical case}

First, we show the existence of a solution to the subcritical equation with negative energy.

LEMMA 8. For each $t>0$, small enough, $\left.\left.\inf _{\|u\|_{H_{2}} \leq t} F_{q}(u)<0, \quad q \in\right] 2, N\right]$.

In fact $F_{q}(t) \leq t^{2}\left(h-t^{q-2} \int_{M} f d v_{g}\right)$, where $h=\max _{M} h(x)$, and since $h<0$, there is $t_{o}>0$ small enough such that $\inf _{\|u\|_{H_{2}} \leq t} F_{q}(u)<0$ for each $\left.t \in\right] 0, t_{o}[$.

Proposition 3. Let $a, h$ be $C^{\infty}$ functions on $M$, with $h$ negative. For every $C^{\infty}$ function, $f$ on $M$ with $\int_{M} f^{-} d v_{g}>0$, there exists a constant $C>0$ which depends only on $\frac{f^{-}}{\int f^{-} d v_{g}}$ such that if $f$ satisfies the following conditions

(1) $|h(x)|<\lambda_{a, f} \quad$ for any $x \in M$

(2) $\frac{\sup f^{+}}{\int f-d v_{g}}<C$

then the subcritical equation

$$
\left.\Delta^{2} u_{q}+\nabla^{i}\left(a \nabla_{i} u_{q}\right)+h u_{q}=f\left|u_{q}\right|^{q-2} u_{q} \quad \text { with } q \in\right] 2, N[
$$

admits a $C^{4, \alpha}$, for some $\alpha \in(0,1)$, solution $u_{q}$ with negative energy. 
Proof. For any $q \in] 2, N\left[\right.$ and $k>0$, let $\mu_{k, q}=\inf _{\|w\|_{q}^{q}=k} F_{q}(w)$. First we remark that if $k$ is close to $0, k>0, \mu_{k, q}<0$ : indeed

$$
\mu_{k, q} \leq F_{q}\left(k^{\frac{1}{q}}\right)=k^{\frac{2}{q}}\left(\int_{M} h d v_{g}-k^{1-\frac{2}{q}} \int_{M} f d v_{g}\right)<0 .
$$

By Proposition 2 the real valued function $k \rightarrow \mu_{k, q}$ is continuous and $\mu_{k, q}$ goes to 0 , when $k \rightarrow 0$. So by Lemma 7 and 8 the function $k \rightarrow \mu_{k, q}$ starts at 0 , takes a negative minimum, say at $k_{q}$, then takes positive values. Let $l_{q}=k_{1, q}=$ $\left[2 \frac{\|h\|_{\infty}+2\left\|a_{+}\right\|_{\infty} C(\sigma)}{\eta \int_{M} f^{-} d v_{g}}\right]^{\frac{q}{q-2}}$ the lower bound of the interval $I_{q}$ given in the proof of Lemma 7 , then

$$
\mu_{k_{q}, q}=\inf _{\|u\|_{q}^{q} \leq l_{q}} F_{q}(u) .
$$

By Proposition 1 the infimum $\mu_{k q, q}$ is attained by a function $v_{q} \in H_{2}$ with $\left\|v_{q}\right\|_{q}^{q}=$ $k_{q}$, so

$$
F_{q}\left(v_{q}\right)=\inf _{\|u\|_{q}^{q} \leq l_{q}} F_{q}(u) .
$$

Now since for any $k_{q} \in I_{q}$, and any $u \in H_{2}$ with $\|u\|_{q}^{q}=k_{q}, F_{q}(u) \geq 0$, it follows that $k_{q}<l_{q}$. So $v_{q}$ is a critical point of $F_{q}$, that is for any $\varphi \in H_{2}$

$$
\begin{gathered}
\int_{M} \Delta v_{q} \Delta \varphi d v_{g}-\int_{M} a \nabla v_{q} \nabla \varphi d v_{g}+ \\
\int_{M} h v_{q} \varphi d v_{g}-\frac{q}{2} \int_{M} f\left|v_{q}\right|^{q-2} v_{q} \varphi d v_{g}=0
\end{gathered}
$$

then $u_{q}=\left(\frac{q}{2}\right)^{\frac{1}{q-2}} v_{q}$ is a weak solution of the subcritical equation with negative energy such that

$$
\left\|u_{q}\right\|_{q}^{q} \leq\left(\frac{q}{2}\right)^{\frac{q}{q-2}} l_{q}
$$

Moreover, arguing as in the proof of the Proposition $1, u_{q} \in C^{4, \alpha}(M)$ with $\alpha \in$ $(0,1)$.

Now we are going to seek a second solution to the subcritical equation with positive energy.

We start by showing that $F_{q}$ with $\left.q \in\right] 2, N[$ satisfies the Palais-Smale condition.

Lemma 9. Let $c$ be a real number, then each Palais-Smale sequence at level $c$ for the functional $F_{q}$ satisfies the Palais -Smale condition.

Proof. First, we show that each Palais-Smale sequence is bounded: we argue by contradiction. Suppose that there exists a sequence $\left(u_{j}\right)$ such that $F_{q}\left(u_{j}\right)$ tends to a finite limit $c, F_{q}^{\prime}\left(u_{j}\right)$ goes strongly to zero and $u_{j}$ to infinite in the $H_{2}$-norm. More explicitly we have

and

$$
\int_{M}\left(\left(\Delta u_{j}\right)^{2}-a\left|\nabla u_{j}\right|^{2}+h u_{j}^{2}\right) d v_{g}-\int_{M} f|u|_{j}^{q} d v_{g} \rightarrow c
$$

$$
\int_{M}\left(\left(\Delta u_{j}\right)^{2}-a\left|\nabla u_{j}\right|^{2}+h u_{j}^{2}\right) d v_{g}-\frac{q}{2} \int_{M} f|u|_{j}^{q-1} v d v_{g} \rightarrow 0
$$

so for any $\varepsilon>0$ there exists a positive integer $A$ such that for every $j \geq A$ we have

$$
\left.\left|\int_{M}\left(\left(\Delta u_{j}\right)^{2}-a\left|\nabla u_{j}\right|^{2}+h u_{j}^{2}\right) d v_{g}-\int_{M} f\right| u\right|_{j} ^{q} d v_{g}-c \mid \leq \varepsilon
$$


and

$$
\left.\left|\int_{M}\left(\left(\Delta u_{j}\right)^{2}-a\left|\nabla u_{j}\right|^{2}+h u_{j}^{2}\right) d v_{g} d v_{g}-\frac{q}{2} \int_{M} f\right| u\right|_{j} ^{q-1} v d v_{g} \mid \leq \varepsilon .
$$

Hence, we get

$$
\left.\left|(q-2) \int_{M}\left(\Delta u_{j}\right)^{2}-a\right| \nabla u_{j}\right|^{2}+h u_{j}^{2} d v_{g}-q c \mid \leq(q+2) \epsilon
$$

and

$$
\left.\left|(q-2) \int_{M} f\right| u_{j}\right|^{q}-2 c \mid \leq 4 \varepsilon
$$

By Lemma 7, we can choose $k$ to be an $L^{q}-$ norm such that

$$
\inf _{\|u\|_{q}^{q}=k} F_{q}(u)>0 \text {. }
$$

Letting $v_{j}=k^{\frac{1}{q}} \frac{u_{j}}{\left\|u_{j}\right\|_{q}}$, we obtain from (4.2) and (4.3) that

$$
\left.\left|(q-2) \int_{M} f\right| v_{j}\right|^{q} d v_{g}-\frac{2 c k^{\frac{2}{q}}}{\left\|u_{j}\right\|_{q}^{2}} \mid \leq 4 \varepsilon \frac{k^{\frac{2}{q}}}{\left\|u_{j}\right\|_{q}^{2}}
$$

and

$$
\begin{gathered}
\left.\left|(q-2) \int_{M}\left(\Delta v_{j}\right)^{2}-a\right| \nabla v_{j}\right|^{2}+h v_{j}^{2} d v_{g}-q c \frac{k^{\frac{2}{q}}}{\left\|u_{j}\right\|_{q}^{2}} \mid \\
\leq(q+2) \epsilon \frac{k^{\frac{2}{q}}}{\left\|u_{j}\right\|_{q}^{2}} .
\end{gathered}
$$

Now since $\left(\left\|v_{j}\right\|_{q}\right)_{j}$ is a bounded sequence, it follows by (4.5) that $\left(v_{j}\right)$ is bounded in $H_{2}$. If $\left\|u_{j}\right\|_{q}$ goes to infinity, it follows from (4.4) and (4.5) that $F_{q}\left(v_{j}\right)$ goes to zero. And since $\left\|v_{j}\right\|_{q}^{q}=k$, we have

$$
\inf _{\|u\|_{q}^{q}=k} F_{q}(u) \leq F_{q}\left(v_{j}\right)
$$

so

$$
\inf _{\|u\|_{q}^{q}=k} F_{q}(u) \leq 0 .
$$

Hence a contradiction. Then the sequence $\left(u_{j}\right)$ is bounded in $H_{2}$. Since $q<N$, the Sobolev injections are compact. Consequently the Palais-Smale condition is satisfied.

Lemma 10. Let $u \in H_{2}$. If the $L_{q}$-norm $\|u\|_{q}^{q}=k$ goes to $+\infty$, then $\mu_{k, q}=$ $\inf _{\|u\|_{q}^{q}=k} F_{q}(u) \rightarrow-\infty$.

Proof. In fact since $\sup _{x \in M} f(x)>0$ let $u$ be a function of class $C^{2}$ with support contained in the open subset $\{x \in M: f(x)>0\}$ of the manifold $M$ such that $\|u\|_{q}^{q}=1$, then $\int_{M} f|u|^{q} d v_{g}>0$ and

$$
F_{q}(k u)=k^{\frac{2}{q}}\left(\int_{M}\left((\Delta u)^{2}-a|\nabla u|^{2}+h u^{2}\right) d v_{g}-k^{\frac{q-2}{q}} \int_{M} f|u|^{q} d v_{g}\right) .
$$

So $\lim _{k \rightarrow+\infty} F_{q}(k u)=-\infty$. 
Proposition 4. Let $a, h$ be $C^{\infty}$ functions on $M$ with $h$ negative. For every $C^{\infty}$ function, $f$ on $M$ with $\int_{M} f^{-}>0$, there exists a constant $C>0$ which depends only on $\frac{f^{-}}{\int f^{-}}$such that if $f$ satisfies the following conditions

(1) $|h(x)|<\lambda_{a, f} \quad$ for any $x \in M$

(2) $\frac{\sup f^{+}}{\int f^{-}}<C$

(3) $\sup f>0$,

then the subcritical equation

$$
\left.\Delta^{2} u+\nabla^{i}\left(a \nabla_{i} u\right)+h u=f|u|^{q-2} u, \quad q \in\right] 2, N[
$$

admits a nontrivial solution of class $C^{4, \alpha}$, for some $\alpha \in(0,1)$, with positive energy.

Proof. By Lemma 7, 8 and 10 the curve $k \rightarrow \mu_{k, q}$ starts at 0 , takes a negative minimum, then takes positive maximum and goes to minus infinite. Mimicking which is done in $([\mathbf{1 1}])$, let $l_{o}$ be an $L^{q}$-norm such that $\mu_{l_{o}, q}$ is a maximum and $l_{1}$, $l_{2}$ two $L^{q}$-norms such that $\mu_{l_{1}, q}=\mu_{l_{2}, q}=0$ with $l_{1}<l_{o}$ and $l_{2}>l_{o}$.

Set

$$
\Gamma=\left\{\gamma \in C\left([0,1], H_{2}\right): \gamma(0)=u_{l_{1}, q}, \gamma(1)=u_{l_{2}, q}\right\},
$$

where $u_{l_{i}, q} \in B_{l_{i}, q}, i=1,2$, are such that $\mu_{l_{i}, q}=F_{q}\left(u_{l_{i}, q}\right)=\inf _{w \in B_{l_{i}, q}} F_{q}(w)$

and

$$
\nu_{q}=\inf _{\gamma \in \Gamma} \max _{t \in[0,1]} F_{q}(\gamma(t)) .
$$

Arguing as in $[\mathbf{1 1}]$, we show that $\nu_{q}$ is a critical level of the functional $F_{q}$ and $\nu_{q} \geq \mu_{l, q}>0$. Consequently the subcritical equation (1.2) admits a weak solution of positive energy. This solution is in fact of class $C^{4, \alpha}$ with $\alpha \in(0,1)$.

Theorem 5 follows from Proposition 3 and 4.

\section{Critical case}

Now, we are going to investigate solutions of the critical equation.

TheOREM 7. Let $a, h$ be $C^{\infty}$ functions on $M$ with $h$ negative. For every $C^{\infty}$ function, $f$ on $M$ with $\int_{M} f^{-}>0$, there exists a constant $C>0$ which depends only on $\frac{f^{-}}{\int f^{-}}$such that if $f$ satisfies the following conditions

(1) $|h(x)|<\lambda_{a}, f \quad$ for any $x \in M$

(2) $\frac{\sup f^{+}}{\int f^{-}}<C$

then the critical equation

$$
\Delta^{2} u+\nabla^{i}\left(a \nabla_{i} u\right)+h u=f|u|^{N-2} u
$$

admits a $C^{4, \alpha}$, for some $\alpha \in(0,1)$, solution $u$ with negative energy.

Proof. For each $q \in(2, N)$, let $u_{q}$ be the solution to the subcritical equation (4.1) given by Proposition $3, u_{q}$ is of negative energy. We have already shown in the proof of Proposition 3 that

$$
\left\|u_{q}\right\|_{q}^{q}=k_{q} \leq l_{q}=\left[2 \frac{\|h\|_{\infty}+2\left\|a_{+}\right\|_{\infty} C(\sigma)}{\eta \int_{M} f^{-} d v_{g}}\right]^{\frac{q}{q-2}}
$$


and since $l_{q}$ goes to $l_{N}=\left[2 \frac{\|h\|_{\infty}+2\left\|a_{+}\right\|_{\infty} C(\sigma)}{\eta \int_{M} f^{-} d v_{g}}\right]^{\frac{4}{n}}$ as $q$ goes to $N,\left(u_{q}\right)$ is bounded in $L^{q}$, so it is in $L^{2}$ and since $u_{q}$ are of negative energy then

$$
\begin{gathered}
\left\|\Delta u_{q}\right\|_{2}^{2} \leq \int_{M} a|\nabla u|^{2} d v_{g}-\int_{M} h u_{q}^{2} d v_{g}+\int_{M} f\left|u_{q}\right|^{q} d v_{g} \\
\leq\left\|a_{+}\right\|_{\infty}\left\|\nabla u_{q}\right\|_{2}^{2}+\|h\|_{\infty}\left\|u_{q}\right\|_{q}^{2}+\|f\|_{\infty}\left\|u_{q}\right\|_{q}^{q} .
\end{gathered}
$$

Now since for any sufficiently $\sigma>0$, there exists a constant $C(\sigma)$ such that

$$
\left\|\nabla u_{q}\right\|_{2}^{2} \leq 2 \sigma\left\|\Delta u_{q}\right\|_{2}^{2}+2 C(\sigma)\left\|u_{q}\right\|_{2}^{2}
$$

we get

$$
\begin{gathered}
\left(1-2 \sigma\left\|a_{+}\right\|_{\infty}\right)\left\|\Delta u_{q}\right\|_{2}^{2} \leq\left(2\left\|a_{+}\right\|_{\infty} C(\sigma)+\|h\|_{\infty}\right)\left\|u_{q}\right\|_{q}^{2}+\|f\|_{\infty}\left\|u_{q}\right\|_{q}^{q} \\
\leq\left(2\left\|a_{+}\right\|_{\infty} C(\sigma)+\|h\|_{\infty}\right) l_{q}^{\frac{2}{q}}+\|f\|_{\infty} l_{q} .
\end{gathered}
$$

So $\left(u_{q}\right)$ is a bounded sequence in $H_{2}$. Consequently $u_{q} \rightarrow v$ weakly in $H_{2}$, up to a subsequence, we have

$$
\begin{gathered}
u_{q} \rightarrow v \text { strongly in } L^{s}(M) \quad \text { for } s<N \\
\nabla u_{q} \rightarrow \nabla v \text { strongly in } L^{2} \\
u_{q}(x) \rightarrow v(x) \quad \text { for } \text { a.e. } x \in M .
\end{gathered}
$$

On the other hand for any $q \in] 2, N\left[, u_{q}\right.$ satisfies, for any $\varphi \in H_{2}$

$$
\begin{gathered}
\int_{M} \Delta u_{q} \Delta \varphi d v_{g}-\int_{M} a \nabla^{i} u_{q} \nabla_{i} \varphi d v_{g}+\int_{M} h u_{q} \varphi d v_{g} \\
=\frac{q}{2} \int_{M} f\left|u_{q}\right|^{q-2} u_{q} \varphi d v_{g}
\end{gathered}
$$

and since the convergence of $\left(u_{q}\right)$ is weak in $H_{2}$, it follows that for any $\varphi \in H_{2}$

$$
\begin{aligned}
& \int_{M} \Delta u_{q} \Delta \varphi d v_{g}-\int_{M} a \nabla^{i} u_{q} \nabla_{i} \varphi d v_{g}+\int_{M} h u_{q} \varphi d v_{g} \\
& \left.\rightarrow \int_{M} \Delta v \Delta \varphi d v_{g}-\int_{M} a \nabla^{i} v \nabla_{i} \varphi\right) d v_{g}+\int_{M} h v \varphi d v_{g} .
\end{aligned}
$$

Moreover since $u_{q}(x) \rightarrow v(x)$ for a.e. $x \in M$ and $\left(u_{q}\right)$ is bounded in $H_{2}$ we have

$$
u_{q}(x)\left|u_{q}(x)\right|^{q-2} \rightarrow v(x)|v(x)|^{N-2} \quad \text { for a.e. } x \in M
$$

and

$$
\left\|u_{q}\left|u_{q}\right|^{q-2}\right\|_{\frac{N}{N-1}}=\left\|u_{q}\right\|_{(q-1) \frac{N}{N-1}}^{q-1} \leq C_{1}\left\|u_{q}\right\|_{N}^{N-1} \leq C\left\|u_{q}\right\|_{H_{2}}^{N-1} .
$$

consequently $\left(u_{q}\right)$ is bounded in $L^{\frac{N}{N-1}}$ and by a well known theorem [1] $u_{q}$ converges weakly to $v$ in $L^{\frac{N}{N-1}}$. Now for any $\varphi \in H_{2} \subset L^{N}$, and any smooth function $f$, $f \varphi \in L^{N}$ ( the dual space of $L^{\frac{N}{N-1}}$ ), then

$$
\int_{M} f\left|u_{q}\right|^{q-2} u_{q} \varphi d v_{g} \rightarrow \int_{M} f|v|^{N-2} v \varphi d v_{g} .
$$

So by $(5.3)$ and $(5.4) u=\left(\frac{N}{2}\right)^{\frac{1}{N-2}} v$ is a weak solution of the critical equation. 
It remains to check that $u \neq 0$. We let

$$
\mu_{k_{q}, q}=\inf _{w \in \bar{B}_{k, q}} F_{q}(w)
$$

where

$$
\bar{B}_{k, q}=\left\{w \in H_{2}(M):\|w\|_{q}^{q} \leq l_{q}\right\} .
$$

By Proposition $1, \mu_{k_{q}, q}$ is attained by by a function $u_{q} \in H_{2}(M)$ with $\left\|u_{q}\right\|=k_{q} \leq$ $l_{q}$ that is $\mu_{k_{q}, q}=F_{q}\left(u_{q}\right)$.

Claim 2. $\mu_{k_{q}, q}$ are uniformly lower bounded, as q goes to $N$.

Indeed, in one hand we have $\mu_{k_{q}, q}<0$ and on the other hand if $\min _{x \in M} a(x) \leq 0$ we obtain

$$
\begin{gathered}
\mu_{k_{q}, q}=F_{q}\left(u_{q}\right) \\
=\left\|\Delta u_{q}\right\|_{2}^{2}-\int_{M} a\left|\nabla u_{q}\right|^{2} d v_{g}+\int_{M} h u_{q}^{2} d v_{g}-\int_{M} f\left|u_{q}\right|^{q} d v_{g} \\
\geq \min _{x \in M} h(x) k_{q}^{\frac{2}{q}}-\max _{x \in M} f^{+}(x) k_{q} .
\end{gathered}
$$

Letting

$$
C_{q}=\max \left(l_{q}, 1\right)
$$

we get

so

$$
\mu_{k_{q}, q} \geq\left(\min _{x \in M} h(x)-\max _{x \in M} f^{+}(x)\right) C_{q}
$$

$$
\lim _{q \rightarrow N} \inf \mu_{k_{q}, q} \geq\left(\min _{x \in M} h(x)-\max _{x \in M} f^{+}(x)\right) C_{N} .
$$

In the case $\min _{x \in M} a(x)>0$, thanks to formula (2.5), we obtain for any sufficiently small $\sigma>0$

$$
\mu_{k_{q}, q} \geq\left(1-\sigma \min _{x \in M} a(x)\right)\left\|\Delta u_{q}\right\|_{2}^{2}+\left(\min _{x \in M} h(x)+\min _{x \in M} a(x) C(\sigma)-\max _{x \in M} f^{+}(x)\right) C_{q}
$$

and taking $\sigma$ small so that $\left(1-\sigma \min _{x \in M} a(x)\right) \geq 0$, we obtain

$$
\mu_{k_{q}, q} \geq\left(\min _{x \in M} h(x)+\min _{x \in M} a(x) C(\sigma)-\max _{x \in M} f^{+}(x)\right) C_{q}
$$

and $\mu_{k_{q}, q}$ are lower bounded as $q \rightarrow N$.

Claim 3. Up to a subsequence we have

$$
\lim _{q \rightarrow N} \mu_{k_{q}, q}=\mu_{k_{N}, N}<0 .
$$

For $q$ close to $N$, we let

$$
0<k<\min \left(l_{q},\left[\frac{\left|\int_{M} h d v_{g}\right|}{2 \int_{M} f^{-} d v_{g}}\right]^{\frac{q}{q-2}}\right) .
$$

Since

$$
\mu_{k_{q}, q}=\inf _{u \in \bar{B}_{k, q}} F_{q}(u)
$$

with

$$
\bar{B}_{k_{q}, q}=\left\{u \in H_{2}:\|u\|_{q}^{q} \leq l_{q}\right\}
$$


we get

$$
\begin{aligned}
\mu_{k_{q}, q} \leq F_{q}\left(k^{\frac{1}{q}}\right)= & k^{\frac{2}{q}}\left(\int_{M} h d v_{g}+k^{1-\frac{2}{q}} \int_{M} f^{-} d v_{g}\right) \\
& \leq \frac{1}{2} k^{\frac{2}{q}} \int_{M} h d v_{g}
\end{aligned}
$$

hence up to a subsequence

$$
\mu_{k_{N}, N}=\lim _{q \rightarrow N} \mu_{k_{q}, q} \leq \frac{1}{2} k^{\frac{2}{N}} \int_{M} h d v_{g}<0 .
$$

Now, we are in position to show that $u=\left(\frac{N}{2}\right)^{\frac{1}{N-2}} v \neq 0$.

Claim 4. The weak solution of the critical equation (5.1) is non trivial.

In fact since $u$ is a solution of the equation (5.1) and the sequence $\left(u_{q}\right)$, of solutions to the subcritical equations, converges weakly to $v$ in $H_{2}$, we have

$$
\begin{gathered}
\frac{N}{2} \int_{M} f|v|^{N}=\left(\|\Delta v\|_{2}^{2}-\int_{M} a|\nabla v|^{2} d v_{g}+\int_{M} h v^{2} d v_{g}\right) \\
\leq \lim \inf _{q \rightarrow N}\left(\left\|\Delta u_{q}\right\|_{2}^{2}-\int_{M} a\left|\nabla u_{q}\right|^{2} d v_{g}+\int_{M} h u_{q}^{2} d v_{g}\right) \\
=\lim \inf _{q \rightarrow N}\left(\frac{2}{q} \int_{M} f\left|u_{q}\right|^{q} d v_{g}\right) .
\end{gathered}
$$

The function $u_{q}$ solution of the subcritical equation achieves the minimum $\mu_{k_{q}, q}=$ $\inf _{u \in \bar{B}_{k}, q} F_{q}(u)$, where $\bar{B}_{k_{q}, q}=\left\{u \in H_{2}:\|u\|_{q}^{q} \leq l_{q}\right\}$.

So

$$
\mu_{k_{q}, q}=F_{q}\left(u_{q}\right)=\left(\frac{q}{2}-1\right) \int_{M} f\left|u_{q}\right|^{q} d v_{g}
$$

and taking account of (5.5) and (5.6), we get

$$
\int_{M} f|v|^{N} d v_{g}<0
$$

hence

$$
u=\left(\frac{N}{2}\right)^{\frac{1}{N-2}} v \neq 0 .
$$

By the bootstrap method and a method imagined by Vaugon see [12], we get that $u$ is of class $C^{4, \alpha}$ for some $\alpha \in(0,1)$.

\section{References}

[1] T.Aubin, Some nonlinear problems in Riemannian geometry, Springer (1998).

[2] M. Benalili, Y. Maliki, Generalized prescribed scalar curvature type equation on a compact manifold of negative scalar curvature. Rocky Mt. J. Math. 37(5) 2007, 1399-1413.

[3] T.P.Branson, Group representations arising from Lorentz conformal geometry, J. Funct. Anal. 74, 1987, 199-291.

[4] F. Bernis, J. Garcia-Azorero, I. Peral, Existence and multiplicity of non trivial solutions in semilinear critical problems of fourth order. Adv. in Differential Equations I (1996) 219-240.

[5] S.Y.A. Chang, On Paneitz operator, A fourth order differential operator in conformal geometry, Harmonic Analysis and Partial Differential Equations, Essays in honor of Alberto P. Calderon, Eds: M. Christ, C. Kenig and C. Sadorsky, Chicago Lectures in Mathematics.

[6] S.Y.A. Chang, P.C. Yang, On a fourth order curvature invariant, Comp. Math. 237, Spectral Problems in Geometry and Arithmetic, Ed. T. Branson, AMS, 1999, 9-28. 
[7] D. Caraffa, Equations elliptiques du quatrième ordre avec exposants critiques sur les variétés riemanniennes compactes. J. Math. Pures Appl., 80, 9 (2001), 941-960.

[8] D.E. Edmunds, D. Fortunato and E. Jannelli, Critical exponents, critical dimensions and the biharmonic operator, Arch.Rational Mech. Anal., 112, (1990), no3, 269-289.

[9] E.Hebey, Sharp Sobolev inequalities of second order, J. Geom. Anal. 13, 1, (2003), 145-162.

[10] S. Paneitz, A quatric conformally covariant differential operator for arbitrary peudoRiemannian manifolds, SIGMA, 4, (2008).

[11] A. Rauzy, Courbures scalaires des variétés d' invariant conforme négatif. Trans. A M S, 347,12 (1995).

[12] R. Van der Vorst, Fourth order elliptic equations, with critical growth, C.R. Acad. Sci. Paris t.320, série I, (1995), 295-299.

[13] H. Yamabe, On the deformation of Riemannian stuctures on compact manifolds, Osaka Math. J. 12, (1960), 21-37.

University Abou-Bekr Belkaïd, Faculty of Sciences Dept. Maths B.P.119 Tlemcen Algeria.

E-mail address: m_benalili@mail.univ-tlemcen.dz 